University of Wollongong

Research Online

Australian Institute for Innovative Materials -

Papers

Australian Institute for Innovative Materials

$1-1-2019$

Clinically amendable, defined, and rapid induction of human brain organoids from induced pluripotent stem cells

\author{
Eva Tomaskovic-Crook \\ University of Wollongong, evatc@uow.edu.au \\ Jeremy Micah Crook \\ University of Wollongong, jcrook@uow.edu.au
}

Follow this and additional works at: https://ro.uow.edu.au/aiimpapers

Part of the Engineering Commons, and the Physical Sciences and Mathematics Commons

Research Online is the open access institutional repository for the University of Wollongong. For further information contact the UOW Library: research-pubs@uow.edu.au 


\title{
Clinically amendable, defined, and rapid induction of human brain organoids from induced pluripotent stem cells
}

\author{
Abstract \\ Human brain organoids provide opportunities to produce three-dimensional (3D) brain-like tissues for \\ biomedical research and translational drug discovery, toxicology, and tissue replacement. Here we \\ describe a protocol for rapid and defined induction of brain organoids from human induced pluripotent \\ stem cells (iPSCs), using commercially available culture and differentiation media and a cheap, easy to \\ handle and clinically approved semisynthetic hydrogel. Importantly, the methodology is uncomplicated, \\ well-defined, and reliable for reproducible and scalable organoid generation, and amendable to principles \\ of current good laboratory practice (cGLP), with the potential for prospective adaptation to current good \\ manufacturing practice (cGMP) toward clinical compliance.

\section{Keywords} \\ amendable, defined, rapid, induction, human, clinically, brain, cells, organoids, induced, pluripotent, stem \\ Disciplines \\ Engineering | Physical Sciences and Mathematics

\section{Publication Details} \\ Tomaskovic-Crook, E. \& Crook, J. M. (2019). Clinically amendable, defined, and rapid induction of human \\ brain organoids from induced pluripotent stem cells. Organoids, 1576 13-22.
}




\title{
Clinically Amendable, Defined, and Rapid Induction of Human Brain Organoids from Induced Pluripotent Stem Cells
}

\author{
Eva Tomaskovic-Crook and Jeremy M. Crook
}

\begin{abstract}
Human brain organoids provide opportunities to produce three-dimensional (3D) brain-like tissues for biomedical research and translational drug discovery, toxicology, and tissue replacement. Here we describe a protocol for rapid and defined induction of brain organoids from human induced pluripotent stem cells (iPSCs), using commercially available culture and differentiation media and a cheap, easy to handle and clinically approved semisynthetic hydrogel. Importantly, the methodology is uncomplicated, well-defined, and reliable for reproducible and scalable organoid generation, and amendable to principles of current good laboratory practice (cGLP), with the potential for prospective adaptation to current good manufacturing practice (cGMP) toward clinical compliance.
\end{abstract}

Keywords Brain organoid, Clinical compliance, Defined, GelMA, Human induced pluripotent stem cells, Hydrogel

\section{Introduction}

The ability to generate three-dimensional (3D) organoids in vitro that recapitulate features of the developing prenatal human brain in utero represents a quantum leap from conventional 2D neural cell culture. Whether they incorporate multiple brain regions as reported by Lancaster and colleagues $[1,2]$ or are comparatively simpler [3], they exhibit a remarkable level of complexity unattainable with cells in 2D. While current methods are unable to precisely recapitulate the complicated patterning and diverse environmental cues found in and experienced by an intact embryo, they are nonetheless rapidly evolving from first-generation systems to more defined, standardized, reproducible, and clinically amendable protocols, vital for experimentation and translational application [4]. In addition, with the need to model development and disease pathogenesis later as well as early in fetal development, extended culture for organoid maturation is necessary.

Here we describe in detail a method to generate human brain organoids for protracted culture and maturation, under defined 
conditions, amendable to cGLP and adaptable to cGMP for clinical compliance. Similar to other protocols for producing organoids described in this volume, a desired level of quality assurance (QA) can be applied for all aspects of the method, including but not limited to the manufacture, procurement, receipt, storage and handling of materials, reagents and iPSCs, as well as cell and organoid culture. While other guidances specifically consider QA for many of the abovementioned processes [5-7], in any event, standardized and reliable protocols are necessary for basic science through applied research and clinical product development.

This chapter details methods that are suitable for routine application, high-quality research, and adaptable for clinical compliance. The culture media used include commercially available cGMP, defined feeder-free and serum-free maintenance medium for human iPSCs, and similarly defined off-the-shelf serum-free medium for initial neural induction and end-stage differentiation medium for organoid formation. Importantly, iPSCs are differentiated, and organoids are maintained on a soft hydrogel substrate produced from photocrosslinkable gelatin methacrylate (GelMA), a derivative of collagen widely used for biomedical applications [8]. Finally, the protocol described is scalable to generate larger numbers of organoids.

\section{Materials}

\section{1 iPSC Culture and Passaging}

\subsection{Preparation of Cast Hydrogel}

1. iPSCs.

2. Corning ${ }^{\circledR}$ Matrigel $^{\circledR}$ (hESC qualified matrix, LDEV-free; Falcon, In Vitro Technologies, Cat. no. FAL354277).

3. Dulbecco's Modified Eagle Medium/Nutrient Mixture F-12 (DMEM/F-12; Gibco, Life Technologies, Cat. no. 11330057).

4. mTeSRl complete kit for hES maintenance (STEMCELL Technologies, Cat. no. 05850).

5. Dulbecco's phosphate-buffered saline, without $\mathrm{Ca}^{2+}$ and $\mathrm{Mg}^{2+}$ (PBS; Sigma Aldrich, Cat. no. D8537).

6. Ethylenediaminetetraacetic acid (EDTA; Sigma Aldrich, Cat. no. E8008).

1. Gelatin methacrylate (GelMA, bloom 300, 80\% degree of substitution, Sigma-Aldrich, Cat. no. 900496) or prepared as described elsewhere (see Note 6).

2. Irgacure 2959 (2-hydroxy-1-[4-(2-hydroxyethoxy)phenyl]-2methyl-1-propanone; CIBA Chemicals, Cat. no. 29891301PS04).

3. Dulbecco's phosphate-buffered saline, without $\mathrm{Ca}^{2+}$ and $\mathrm{Mg}^{2+}$ (PBS; Sigma Aldrich, Cat. no. D8537). 
4. 100\% ethanol (Chem-Supply, Cat. no. EA043).

5. DMEM/F-12 medium (Gibco, Life Technologies, Cat. no. 11330057).

2.3 Neural Induction of iPSCs: Generation of Neural Progenitor Cells

2.4 Differentiation of iPSC-Derived Neural Progenitor Cells

\subsection{General}

\section{Equipment}

1. STEMdiff Neural Induction Medium (STEMCELL Technologies, Cat. no. 05835).

1. Neurobasal Medium (Gibco, Life Technologies, Cat. no. 21103049).

2. DMEM/F-12 medium (Gibco, Life Technologies, Cat. no. 11330057).

3. NeuroCult SMI neuronal supplement (STEMCELL Technologies, Cat. no. 05711).

4. N2 supplement-A (STEMCELL Technologies, Cat. no. 07152).

5. Brain-derived neurotrophic factor (BDNF; Cat. no. AF-450-02; PeproTech, Lonza).

6. Tris-HCl (Sigma-Aldrich, Cat. no. T5941).

7. Human serum albumin (Sigma-Aldrich, Cat. no. A9511).

8. L-Glutamine (200 mM; Gibco, Life Technologies, Cat. no. 25030081).

1. 6-well and 24-well tissue culture plates (Costar, Sigma-Aldrich).

2. Pipetman (micropipette) and tips.

3. Pipet-Aid (motorized pipette) and serological pipettes.

4. $15 \mathrm{~mL}$ conical tubes (Corning, Cat. no. 430052).

5. Parafilm M laboratory film (Edwards Group, Cat. no. PM992).

6. Class 2 Biological Safety Cabinet (biosafety cabinet).

7. Humidified $5 \% \mathrm{CO}_{2}$ in air incubator maintained at $37{ }^{\circ} \mathrm{C}$.

8. Dry $37^{\circ} \mathrm{C}$ incubator.

9. Inverted phase-contrast microscope.

10. Low-speed centrifuge.

11. Liquid nitrogen storage tank.

12. $-80{ }^{\circ} \mathrm{C}$ freezer.

13. Refrigerator.

14. UV light source (365 $\mathrm{nm}$ range).

15. Luminometer. 
3 Methods

\subsection{Routine iPSC Culture and Passaging}

Reagent preparation and cell culture work should be performed in a biosafety cabinet unless otherwise specified. All media and reagents should remain sterile. Incubations and culturing should be performed in a $37{ }^{\circ} \mathrm{C}$ incubator with a humidified atmosphere of $5 \%$ $\mathrm{CO}_{2}$ in air. Centrifuge steps are performed at room temperature (RT).

iPSCs are cultured as feeder-free cultures as in the method outlined below (see Note 1). Importantly, a good quality iPSC culture with low spontaneous differentiation is essential for efficient neural induction.

1. Prepare Corning ${ }^{\circledR}$ Matrigel ${ }^{\circledR}$ matrix as per the manufacturer's instructions and store frozen aliquots at $-80^{\circ} \mathrm{C}$ (see Note 2 ).

2. Thaw Matrigel aliquot at $4{ }^{\circ} \mathrm{C}$ in refrigerator, $2 \mathrm{~h}$ before use.

3. Transfer contents of thawed aliquot to $6 \mathrm{~mL}$ DMEM/F-12 media prechilled to $4{ }^{\circ} \mathrm{C}$ in a sterile $15 \mathrm{~mL}$ conical tube. Gently pipet up and down with a serological pipette to mix contents.

4. Immediately add $1 \mathrm{~mL}$ diluted Matrigel matrix per well of 6-well plate. Swirl and rock plate back and forth to evenly coat the surface of the well. Seal plate edges with Parafilm, wrap in foil, and transfer to a level shelf within a refrigerator to incubate overnight at $4{ }^{\circ} \mathrm{C}$.

5. The following day, carefully remove Matrigel matrix with serological pipette (without scratching bottom of well) and add $1 \mathrm{~mL}$ per well DMEM/F-12 to rinse.

6. Remove and add $2 \mathrm{~mL}$ DMEM/F-12 to cover each coated well. Coated wells may be used immediately on the same day or prepared up to 2 weeks in advance. If to be stored at $4{ }^{\circ} \mathrm{C}$ in a refrigerator, seal plate edges with Parafilm and wrap in foil before storage.

7. Prepare iPSC culture medium (mTeSRl) as per the manufacturer's instructions and store at $4{ }^{\circ} \mathrm{C}$ in refrigerator (see Note 3).

8. Monitor iPSCs daily by viewing under phase-contrast microscope to ensure appropriate timing of passaging and removal of areas of differentiation prior to passage. iPSCs are passaged prior to colonies becoming confluent (approximately 60-75\% confluency across well) and when center of each colony has become dense, but not overgrown. For routine passaging, cells undergo a 1:6 to $1: 12$ split ratio.

9. When iPSC colonies are ready to passage, prewarm iPSC culture media, DMEM/F-12 media, PBS, and EDTA solution to 
$37^{\circ} \mathrm{C}$ in dry incubator $\mathrm{l}$ h before use. Prior to use, allow stored Matrigel-coated plates to equilibrate to room temperature for $30 \mathrm{~min}$.

10. Just before dissociating cells for passaging, aspirate DMEM/F12 from Matrigel-coated wells and add $2 \mathrm{~mL}$ prewarmed iPSC culture media to each well of 6-well plate and place in humidified $37^{\circ} \mathrm{C} / 5 \% \mathrm{CO}_{2}$ incubator until ready to seed.

11. Aspirate spent culture media from wells to be passaged. Wash each well briefly with $1 \mathrm{~mL}$ PBS and aspirate PBS solution.

12. To dissociate iPSCs, add $1 \mathrm{~mL} 0.02 \%$ EDTA (see Note 4 ), and incubate for $2 \mathrm{~min}$ in $37{ }^{\circ} \mathrm{C} / 5 \% \mathrm{CO}_{2}$ incubator.

13. Once detachment of edge of colonies viewed under phasecontrast microscope has been confirmed, carefully aspirate EDTA solution.

14. With a $5 \mathrm{~mL}$ serological pipette, immediately add $3 \mathrm{~mL}$ prewarmed DMEM/F-12 media to a well. While slowly dispensing the contents, scrape the tip of the serological pipette over the surface of the well to dislodge the cells.

15. Repeat one to two times to dislodge the iPSC colonies, but do over-pipette cell suspension to ensure dissociation of cell aggregates (approximately 10-20 cells) rather than single cells. Transfer dissociated cell aggregates to a $15 \mathrm{~mL}$ conical tube.

16. For routine passaging, plate three wells at a range of seeding densities to enable optimal passaging approximately every 3-4 days in culture. For example, depending on the iPSC line, cells may be seeded at a 1:6, 1:10, and 1:20 split ratio. For instance, to achieve a 1:6 split ratio, add $0.5 \mathrm{~mL}$ of dissociated cell aggregates per well of a 6-well plate prepared earlier (see Sect. 3.1, Step 10), containing $2 \mathrm{~mL}$ prewarmed iPSC culture media, and stored in humidified $37^{\circ} \mathrm{C} / 5 \% \mathrm{CO}_{2}$ incubator.

\subsection{Preparation of Cast GelMA Hydrogel}

GelMA hydrogels are prepared by directly casting into wells of standard 24-multiwell culture plates (see Note 5 ).

1. Freshly prepare $5-10 \%(\mathrm{w} / \mathrm{v})$ GelMA (see Note 6) with $0.5 \%$ Irgacure 2959 in PBS solution (see Note 7). Dissolve by heating to $37^{\circ} \mathrm{C}$ for $1 \mathrm{~h}$ and mix well.

2. Add $0.2 \mathrm{~mL}$ prepolymerized solution per well of 24 -well plate for casting of hydrogel. Ensure an even coating of the well by rocking plate back and forth.

3. Immediately crosslink the prepolymerized solution by exposure of each well to $100 \mathrm{~mW} / \mathrm{cm}^{2}$ UV light $(365 \mathrm{~nm})$ for $60 \mathrm{~s}$ via an optical fiber connected to a Dymax BlueWave 75 UV spot lamp (see Note 8 ). 
4. Immediately following polymerization, add $1 \mathrm{~mL}$ PBS to each well.

5. Soak hydrogel for $2 \mathrm{~h}$ at $4{ }^{\circ} \mathrm{C}$. Rinse hydrogel twice in PBS.

6. Continue soaking of hydrogel overnight at $4{ }^{\circ} \mathrm{C}$.

7. The following day, replace PBS solution with $1 \mathrm{~mL}$ DMEM/ F12 media per well.

8. Store pre-made cast hydrogels at $4{ }^{\circ} \mathrm{C}$ in refrigerator. Seal plate edges with Parafilm and wrap in foil before storage.

3.3 Surface Seeding of iPSCs Onto Hydrogel and Generation of Neural Progenitor Cells
iPSCs are seeded onto cast hydrogel as dissociated cells in mTeSRl (see Note 5). Colonies are allowed to attach and expand, before neural induction using STEMdiff Neural Induction Medium (Fig. 1).

1. Prewarm DMEM/F-12 and mTeSRl media for 1 h at $37^{\circ} \mathrm{C}$ in dry incubator before use.

2. Pre-made cast hydrogels stored at $4{ }^{\circ} \mathrm{C}$ are equilibrated to room temperature for $1 \mathrm{~h}$.

3. Carefully remove DMEM/F-12 media from wells without disturbing cast hydrogel from well.

4. Wash hydrogel in each well of 24 -well plate with $1 \mathrm{~mL}$ prewarmed DMEM/F-12 media. Allow to incubate for at least $15 \mathrm{~min}$ in a $37^{\circ} \mathrm{C} / 5 \% \mathrm{CO}_{2}$ incubator.

5. Just before dissociating cells for passaging, aspirate DMEM/F12 from wells, and add $1 \mathrm{~mL}$ prewarmed iPSC culture media to each well of 24-well plate containing cast hydrogel, and place in humidified $37{ }^{\circ} \mathrm{C} / 5 \% \mathrm{CO}_{2}$ incubator until ready to seed.

6. iPSCs are dissociated with EDTA as described in Sect. 3.1 for routine iPSC cell culture and dissociation.

7. Following dissociation, using a $2 \mathrm{~mL}$ serological pipette, slowly pipette cell aggregate suspension up and down twice, and seed dissociated cell aggregates at one drop per well (approximately $50 \mu \mathrm{L})$.

8. Return 24-well plate to humidified $37^{\circ} \mathrm{C} / 5 \% \mathrm{CO}_{2}$ incubator. Distribute cells across the surface of the hydrogel by moving plate side to side and front and back (at right angles).

9. Leave plates undisturbed overnight before confirming attachment of cells the following day.

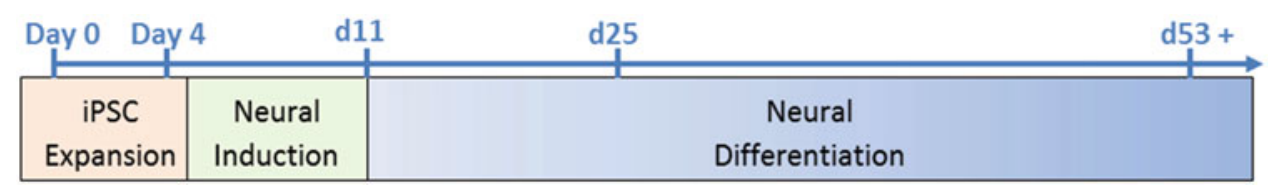

Fig. 1 Schematic for neural induction and differentiation of iPSCs seeded onto hydrogel 
10. Replenish mTeSRI media cells every 2 days. During media change, ensure the cells are minimally dislodged by slowly pipetting to the side of the well.

11. Before and after media change, check attachment of cells to hydrogel surface and cell clustering by phase-contrast microscopy. Return 24 -well plate to humidified $37{ }^{\circ} \mathrm{C} / 5 \% \mathrm{CO}_{2}$ incubator after media change.

12. On the fourth day after seeding, iPSC colonies are transitioned to Neural Induction Media (see Note 9). Prewarm STEMdiff Neural Induction Media for $1 \mathrm{~h}$ at $37{ }^{\circ} \mathrm{C}$ in dry incubator before use.

13. Carefully remove spent media and add $1 \mathrm{~mL}$ STEMdiff Neural Induction Media to each well. Observe cells with phase-contrast microscopy. Return 24-well plate to humidified $37^{\circ} \mathrm{C} / 5 \% \mathrm{CO}_{2}$ incubator.

14. To maintain cultures, perform a half media change every $2-3$ days by removal of $0.5 \mathrm{~mL}$ of spent media and addition of $0.5 \mathrm{~mL}$ STEMdiff Neural Induction Media to each well. Observe cells with phase-contrast microscopy. Return 24-well plate to humidified $37^{\circ} \mathrm{C} / 5 \% \mathrm{CO}_{2}$ incubator.

3.4 Neural Differentiation of iPSCGenerated Neural Progenitor Cells
Following neural stem cell induction and proliferation, iPSC-neural progenitor cells are transitioned to undergo neural differentiation (Fig. 1).

1. Prepare Neural Differentiation Medium (e.g., $50 \mathrm{~mL}$; see Note 10):

- Neurobasal Medium: $25 \mathrm{~mL}$

- DMEM/F-12 medium: $25 \mathrm{~mL}$

- NeuroCult SMl neuronal supplement: $500 \mu \mathrm{L}$ (1\% final conc.)

- $\quad \mathrm{N} 2$ supplement-A: $250 \mu \mathrm{L}(0.5 \%$ final conc.)

- L-Glutamine: $500 \mu \mathrm{L}(1 \times$ final conc. $)$

- $\operatorname{BDNF}(250 \mu \mathrm{g} / \mathrm{mL}$ stock; see Note 11$): 10 \mu \mathrm{L}(50 \mathrm{ng} / \mathrm{mL}$ final concentration).

2. Prewarm Neural Differentiation Medium at $37^{\circ} \mathrm{C}$ in dry incubator, 1 h before use (see Note 12).

3. To transition iPSC-neural progenitor cells to undergo neural differentiation, remove spent Neural Induction Media from each well. Be particularly careful not to dislodge the cells or the hydrogel.

4. Slowly add $1 \mathrm{~mL}$ prewarmed Neural Differentiation Medium to each well. Return 24-well plate to humidified $37^{\circ} \mathrm{C} / 5 \% \mathrm{CO}_{2}$ incubator. 
5. Feed cultures every $3-4$ days with a half media change to replenish media. Perform a half media change by removal of $0.5 \mathrm{~mL}$ of spent media and addition of $0.5 \mathrm{~mL}$ Neural Differentiation Medium to each well. Due to evaporation of media over time, it may be necessary to remove a smaller amount of spent media (roughly half the residual volume of the media in well). Replace with $0.5 \mathrm{~mL}$ Neural Differentiation Medium.

6. Monitor cultures daily and inspect morphology (semitransparent microspikes on outer surface of neurosphere) and acidity of media (change media before reaching orange/yellow color). Continue culture until desired stage of development is reached.

\section{Notes}

1. For complete details of culture, adaptation, and expansion of pluripotent stem cells, refer to Brehm and Ludwig [9].

2. Refer to supplied Certificate of Analysis for Corning ${ }^{\circledR}$ Matrigel $^{\circledR}$ matrix to prepare appropriate aliquot size using a dilution factor to achieve $6 \mathrm{~mL}$ total volume of diluted matrix.

3 . The iPSC colonies are routinely propagated as colonies in mTeSRl media. Thaw frozen mTeSRl supplement overnight at $4{ }^{\circ} \mathrm{C}$. Once thawed, prepare aliquots of $\mathrm{mTeSRl}$ supplement in working volumes and store at $-20{ }^{\circ} \mathrm{C}$. Do not refreeze. To ensure stability of components, keep mTeSRl medium for up to 2 weeks at $4{ }^{\circ} \mathrm{C}$. Other feeder-independent culture media systems may also be employed.

4. Although EDTA $(0.02 \% \mathrm{w} / \mathrm{v}$ in PBS; $0.5 \mathrm{mM})$ method of iPSC dissociation is described, iPSCs may also be dissociated using other reagents, such as Versene $(0.02 \%$, Lonza, Cat. no. 17-711E).

5. High-throughput production of organoids can be achieved by seeding and differentiating iPSCs onto either hydrogel cast into wells of standard plastic-multiwell culture plates or custom bioprinted hydrogel-multiwell plates.

6. A commercial supplier of GelMA is provided. However, depending on the required application, GelMA monomer can be produced from gelatin (Sigma-Aldrich) with tunable modification of the methacrylation degree and gel concentration; refer to Nichol et al. and O'Connel et al. [10, 11].

7. Alternatively, a 10\% (w/v) Irgacure 2959 stock solution dissolved in $100 \%$ ethanol can be prepared and stored at RT, wrapped in foil to keep dark. Dilute to final concentration of $0.5 \%(\mathrm{w} / \mathrm{v})$ in GelMA monomer solution before polymerization. Depending on the application, other photoinitiators, for 
example, VA-086 (2, 2'-azobis[2-methyl- $N$-(2-hydroxyethyl) propionamide], Wako Pure Chemical Industries), can be used.

8. Apply UV light through a covered tissue culture plate to maintain sterility. To ensure consistent application of energy of UV light to polymer solution, measure intensity of light using a luminometer. Expose each well separately by positioning optical fiber directly over each well.

9. Thaw frozen STEMdiff Neural Induction Medium at room temperature or overnight at $4{ }^{\circ} \mathrm{C}$. To ensure stability of components, keep STEMdiff Neural Induction Medium for up to 2 weeks at $4{ }^{\circ} \mathrm{C}$. Once thawed, prepare aliquots in working volumes and store at $-20{ }^{\circ} \mathrm{C}$. Do not refreeze.

10. To ensure stability of components, prepare sufficient working volume of Neural Differentiation Medium for 2-3 weeks of neural differentiation at any one time. Store at $4{ }^{\circ} \mathrm{C}$ for $2-3$ weeks.

11. To prepare $250 \mu \mathrm{g} / \mathrm{mL}$ BDNF stock solution, dissolve $100 \mu \mathrm{g}$ vial of BDNF in $0.4 \mathrm{~mL}$ filter-sterilized $5 \mathrm{mM}$ Tris- $\mathrm{HCl}$ containing $0.5 \%$ human serum albumin, $\mathrm{pH}$ 7.6. Store $10 \mu \mathrm{L}$ aliquots of $250 \mu \mathrm{g} / \mathrm{mL}$ BDNF stock solution at $-80^{\circ} \mathrm{C}$. Do not freeze/thaw vials.

12. Immediately after use, return tube of Neural Differentiation Medium to $4{ }^{\circ} \mathrm{C}$ to preserve stability of components.

\section{Acknowledgment}

The authors wish to acknowledge funding from the Australian Research Council (ARC) Centre of Excellence Scheme (CE140100012).

\section{References}

1. Lancaster MA, Knoblich JA (2014) Generation of cerebral organoids from human pluripotent stem cells. Nat Protoc 9:2329-2340

2. Lancaster MA, Renner M, Martin CA, Wenzel D, Bicknell LS, Hurles ME et al (2013) Cerebral organoids model human brain development and microcephaly. Nature 501:373-379

3. Li Y, Muffat J, Omer A, Bosch I, Lancaster MA, Sur M et al (2017) Induction of expansion and folding in human cerebral organoids. Cell Stem Cell 20:385-396 e3

4. Lindborg BA, Brekke JH, Vegoe AL, Ulrich CB, Haider KT, Subramaniam S et al (2016) Rapid induction of cerebral organoids from human induced pluripotent stem cells using a chemically defined hydrogel and defined cell culture medium. Stem Cells Transl Med 5:970-979

5. Crook JM, Ludwig TE (eds) (2017) Stem cell banking: concepts and protocols. methods in molecular biology, vol 1590. Springer, New York

6. Andrews PW, Baker D, Benvinisty N, Miranda B, Bruce K, Brustle O et al (2015) Points to consider in the development of seed stocks of pluripotent stem cells for clinical applications: international stem cell banking initiative (ISCBI). Regen Med 10:1-44

7. International Stem Cell Banking I (2009) Consensus guidance for banking and supply of human embryonic stem cell lines for research purposes. Stem Cell Rev 5:301-314 
8. Yue K, Trujillo-de Santiago G, Alvarez MM, Tamayol A, Annabi N, Khademhosseini A (2015) Synthesis, properties, and biomedical applications of gelatin methacryloyl (GelMA) hydrogels. Biomaterials 73:254-271

9. Brehm JL, Ludwig TE (2017) Culture, adaptation, and expansion of pluripotent stem cells. In: Crook JM, Ludwig TE (eds) Stem cell banking: concepts and protocols. Methods in molecular biology, vol 1590. pp 139-150
10. Nichol JW, Koshy ST, Bae H, Hwang CM, Yamanlar S, Khademhosseini A (2010) Cellladen microengineered gelatin methacrylate hydrogels. Biomaterials 31:5536-5544

11. O'Connell CD, Di Bella C, Thompson F, Augustine C, Beirne S, Cornock R et al (2016) Development of the Biopen: a handheld device for surgical printing of adipose stem cells at a chondral wound site. Biofabrication 8:015019 